\section{Trial of labor after cesarean}

\section{outcomes with excessive}

\section{gestational weight gain}

\author{
Sophia Halassy* and Angela Marras
}

Department of Obstetrics \& Gynecology, Ascension Providence Hospital/Michigan State University College of Human Medicine, Southfield, MI, USA

\section{Summary}

The success of vaginal birth after cesarean section (VBAC) has been correlated with a variety of maternal characteristics such as age, ethnicity, and body mass index (BMI, calculated as weight in kilograms divided by height in meters squared). The patient's BMI just prior to delivery has been shown to be a better prognostic indicator of success than pre-pregnancy BMI. Gestational weight gain was previously associated with a decreased rate of successful VBAC. More recent research has not supported such an association. The objective of our study was to further validate these findings with a larger population.

We performed a retrospective cohort study of women undergoing trial of labor after cesarean (TOLAC) at our institution from January 2010 to December 2019. Women were divided into three groups based on weight gain in pregnancy as compared to the American College of Obstetrics and Gynecology (ACOG) recommendations (i.e. < 25 pounds (Ibs), 25-35 lbs, > $35 \mathrm{lbs}$ ). We further subdivided the pregnancies between term and preterm deliveries. The primary outcome was a successful VBAC. Of 1087 patients attempting a TOLAC, $772(71 \%)$ were successful and $315(29 \%)$ failed VBAC. When grouped according to ACOG weight gain recommendations, 303 (31\%) women were below ACOG guidelines, 318 (33\%) met guidelines, and 339 (35\%) exceeded guidelines. There was no difference in the rate of VBAC success among the three groups. When counseling patients, providers should still promote healthy dietary habits but should not correlate excess weight gain with chance of TOLAC success.

\author{
More Information \\ *Address for Correspondence: Dr. Sophia \\ Halassy, Department of Obstetrics \& Gynecology, \\ Ascension Providence Hospital, Michigan State \\ University, College of Human Medicine, 16001 \\ West 9 Mile Rd, Southfield, MI 48075, \\ Tel: (248)849-3401; Fax: (248)849-5398; \\ Email: shalassy@gmail.com; \\ halassy@ascension.org \\ Submitted: 28 April 2020 \\ Approved: 05 May 2020 \\ Published: 06 May 2020
}

How to cite this article: Halassy S, Marras A. Trial of labor after cesarean outcomes with excessive gestational weight gain. Clin J Obstet Gynecol. 2020; 3: 039-042.

DOI: 10.29328/journal.cjog.1001048 ORCiD: orcid.org/0000-0001-9430-407X

Copyright: @ 2020 Halassy S, et al. This is an open access article distributed under the Creative Commons Attribution License, which permits unrestricted use, distribution, and reproduction in any medium, provided the original work is properly cited.

Keywords: Gestational weight gain; Vaginal birth after cesarean section; Trial of labor after cesarean section

Check for updates

OPEN ACCESS

\section{Introduction}

As the rate of obesity in the United States has increased substantially over the last decade, more research has focused on the implications of obesity and pregnancy. At this time, the National Academy of Medicine has published weight gain recommendations for pregnancy with a 25 to 35 pound weight gain range for normal weight women (body mass index (BMI) of 18.5-24.9), versus an 11 to 20 pound weight gain for patients categorized as obese (BMI of 30 or greater) [1]. These recommendations are based on the premise that the amount of weight gain during pregnancy can affect both the immediate and future health of the woman and her infant. In 2010, Margerison Zilko, et al. demonstrated that there was an association between gestational weight gain and large for gestational age (LGA) infants. The authors further demonstrated an association between increased weight gain and risk of cesarean section [2.3]. Maternal obesity poses an increased risk for complications after cesarean section and often requires increased anesthetic, intra-operative, and post-operative surveillance [4]. As such, proper prepregnancy counseling is essential to optimizing the antenatal, intrapartum, and postpartum care [5]. Appropriate dietary counseling during prenatal care has been shown to be effective in decreasing the total amount of weight gain in pregnancy and decreasing long-term postpartum weight retention. Overall, this has not yet been demonstrated to have any conclusive benefit on infant and maternal health [6].

When caring for patients with a history of cesarean section, a thorough discussion of the risks and benefits of a trial of labor is essential. Counseling at prenatal appointments should include discussion about such benefits as avoidance of abdominal surgery with lower rates of hemorrhage, thromboembolism and infection. These women typically also have a shorter recovery than women opting for repeat cesarean delivery [7-10]. The most commonly associated risk of vaginal birth after cesarean section (VBAC) has been 
uterine rupture. Predictive models have been implemented in an attempt to predict the success of trial of labor after a history of cesarean delivery (TOLAC). However, none have been widely used due to predictive limitations. These models, which aid in appropriately counselling a patient about her decision, commonly incorporate maternal age, BMI, race, prior vaginal delivery, indication for prior cesarean delivery, and history of VBAC.

A patient's BMI just prior to delivery has been demonstrated to be a better prognostic indicator of success as opposed to the maternal BMI just prior to pregnancy itself [11]. Weight gain in between subsequent pregnancies has been negatively correlated with the success rate [12]. The gestational weight gain and its association with success of VBAC has been previously studied with differing outcomes. One such study was published in 2005 by Juhasz et al. and demonstrated that excessive weight gain during pregnancy and obesity decreased VBAC success [13]. Later, Hibbard et al. in 2006 concluded that increased BMI correlates with TOLAC outcome, and morbidly obese women were at higher likelihood of VBAC failure [14]. In addition, increased BMI had a greater composite morbidity and neonatal injury. This study included almost 30,000 patients and was well powered. However, a recent publication in the American Journal of Perinatology demonstrated no such association [15]. This study included 614 patients, and did not show a statistically significant difference in outcomes. Their post-hoc power analysis showed that they were powered to detect a $12.5 \%$ difference in TOLAC success rates. The object of our study was to further verify these findings with a larger study population and to determine whether gestational weight gain truly had an effect on TOLAC outcome.

\section{Methods}

We conducted a retrospective data analysis of selected patients who had a history of a prior single low transverse cesarean section between the years of 2010 and 2019. We included all patients who attempted TOLAC between the ages of 18-55 and who were admitted to the labor and delivery unit at our institution. Patient care (antepartum, delivery and postpartum) is primarily carried out by Obstetrics \& Gynecology residents and private group attendings. Patient charts were abstracted for maternal age, medical \& obstetric history, and intrapartum and postpartum events. Inclusion criteria consisted of all women admitted to the labor and delivery unit who a) underwent a trial of labor at greater than or equal to 32 weeks of gestation, b) had a vertex, singleton gestation, c) and a history of a single prior low transverse cesarean section. Exclusion criteria included: a) women with a current antepartum intrauterine fetal demise, b) gestational age less than 32 weeks, and c) women without recorded gestational weight gain. From these data, we subdivided the patients based on weight gain with the first category being less than 25 pounds of gestational weight gain, the second between 25 to 35 pounds and the third being greater than 35 pounds.
Data analysis was performed using IBM SPSS (Version 23) statistical software [16] with Chi Square analysis to determine associations between discrete variables and the Student's T-test for continuous variables. A p-value less than 0.05 was considered significant.

\section{Results}

There were a total of 1334 patients that underwent TOLAC between 2010-2019. After application of inclusion and exclusion criteria, 950 patients remained for evaluation. Of these, 887 (92.3\%) were term (defined as gestational age (GA) greater than or equal to 37 weeks of gestation) and 73 $(7.7 \%)$ were preterm. In the preterm population, $19(26 \%)$ of patients gained more than the recommended 25 to $35 \mathrm{lbs}$ weight gain and there was no statistical difference between successful VBAC and failed TOLAC for this population. Patients with successful VBAC were just as likely to be in any of the weight gain groups. Similarly failed TOLAC patients were not more prevalent in any of the three weight gain groups.In the term population as well, $320(36.5 \%)$ of patients gaining more than the recommended GWG were no more likely to have a successful versus failed TOLAC. A quarter of the patients (24.9\%) had a history of previous VBAC with the majority of those $(65.8 \%)$ having had only one. There was no difference in the success of TOLAC among the three gestational weight gain categories (Figures 1,2).

A total of 667 (70.2\%) of patients had a successful VBAC, whereas 283 (29.3\%) were unsuccessful. There was no demonstrable association between gestational weight gain and the likelihood of successful VBAC.

\section{Discussion}

Our study found no association between the amount of gestational weight gain and rate of successful TOLAC. Even

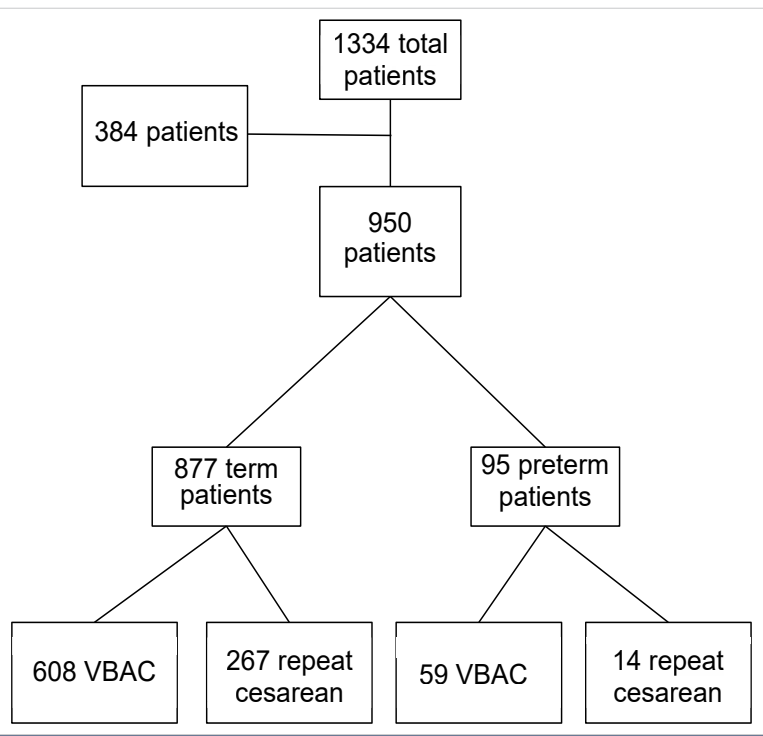

Figure 1: Diagram demonstrating studied patient populations. (term = greater than or equal to 37 weeks of gestation, preterm=greater than or equal to 32 weeks of gestation and less than 37 weeks of gestation, VBAC=vaginal birth after cesarean) 


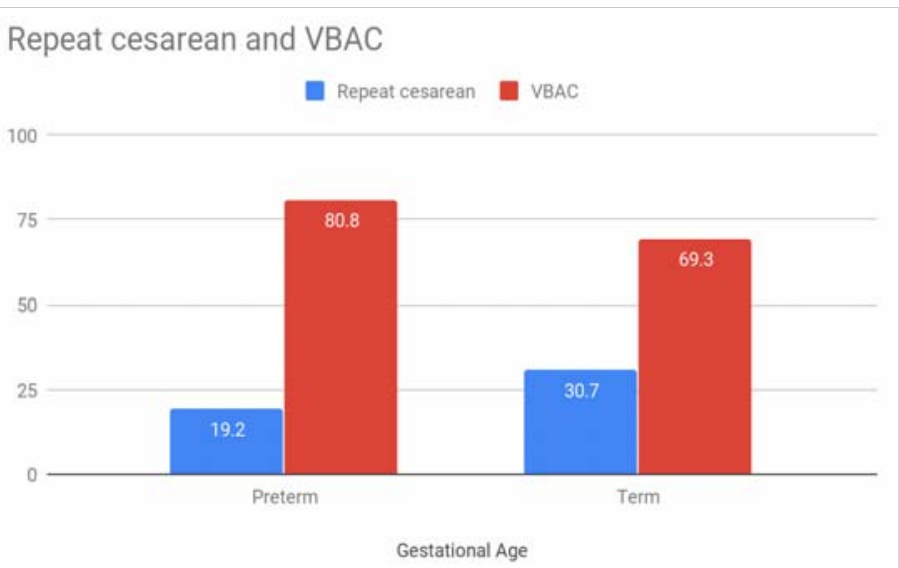

Figure 2: Histogram demonstrating percent of successful (red) and unsuccessful (blue) trials of labor after cesarean section for the preterm (left; gestational age greater than or equal to 32 weeks of gestation and less than 37 weeks of gestation) and term (right; gestational age greater than or equal to 37 weeks) patient populations.

when further subdivided for recommended weight gain in pregnancy, no association was demonstrated. These results confirm the findings proposed by Mei, et al. in 2019 [15], though this study had limited power and included only 614 women. Our results equally contrasted with previously published data $[5,12,13]$ by failing to demonstrate any significance between the failed TOLAC group and gaining more than the recommended GWG. A possible hypothesis for the difference in findings includes the earlier study dates $(\sim 20$ years ago), when TOLAC may not have been encouraged and less was known about risks \& benefits (i.e. "once a c-section, always a c-section").

The prevalence of cesarean delivery is high, with the National Center for Health Statistics (NCHS) noting a 31.9\% rate in the United States during the year of 2018 [17]. For women with a previous cesarean delivery, the American Pregnancy Association estimates 4 out of 5 (80\%) of women attempting VBAC are successful. During prenatal care, a thorough discussion with patients regarding risks and benefits of TOLAC is essential to proper care. According to the Brost, et al. while comparing elective repeat cesarean delivery to TOLAC in term patients, there is a greater maternal risk of infectious morbidity, surgical injury, blood transfusion, and uterine rupture associated with TOLAC. This in contrast to a higher maternal risk of hysterectomy and maternal death (though very minimal) in those with elective repeat surgery [5]. On review of neonatal morbidity, the greatest difference in outcomes appears to be the risk of respiratory morbidity, neonatal and perinatal mortality in those born by TOLAC. Of course, appropriate candidates should be chosen, taking into account demographic and obstetric history, in comparing the risks to the benefits. Other factors which need to be taken into account are the longer length of hospital stay and hospital costs for those undergoing surgery. For obese patients, surgical risks from the procedure itself, anesthesia, and postoperative healing must be discussed. Ultimately, the choice should be made in accordance with patient preference.
While our data do not demonstrate increased risk of repeat cesarean section with greater gestational weight gain, promoting proper heath maintenance is still of great importance, as greater weight prior to TOLAC attempt is associated with decreased success [14]. The ante- and postpartum maternal and neonatal effects of excessive weight gain should still be considered [12] and should remain the mainstay of care when educating and counseling any patient, regardless of previous cesarean section history. Proper counseling regarding gestational weight gain appears to have a greater impact on patients prior to establishment of pregnancy itself [6].

Our large percentage of patients who had excessive weight gain (in excess of 25-35 pounds for a normal BMI woman) demonstrates room for improvement. A limitation of this study was the subjective recall of gestational weight gain upon presentation to Labor \& Delivery. This was not objectively measured and instead given by the patient on admission and is therefore somewhat unreliable. Other limitations include the risk of possible selection bias with our research being conducted retrospectively. Additionally, as expected, gestational weight gain increases with progressing gestational age. However, only $8.7 \%$ of our patients were classified as preterm. The average gestational age for our studied population was 39 weeks. A limiting factor for the study was the lack of interface between the Centricity Perinatal System ${ }^{\mathrm{TM}}$ and the Cerner Electronic Health Record platform ${ }^{\circledR}$. This limited the availability of demographic data available on our study patients. We must also consider that ACOG's recommendation for GWG differs with pre-pregnancy weight, and our study did not take this into account, given lack of available prepregnancy weight data. The largest strength of this study includes the large population of patients, giving our results greater power and increased reliability.

\section{Conclusion}

An important role of an obstetrician is to guide, counsel, and educate the pregnant population to optimize and benefit the birth outcome. Healthy lifestyle and diet should be discussed at each prenatal visit. For those with a history of cesarean section, risks and benefits of TOLAC should be discussed, keeping in mind the patient's best interest and likelihood of success. While excessive gestational weight gain does not appear to have an effect on TOLAC success, adherence to recommend gestational weight gain levels should still be encouraged to improve maternal and neonatal outcomes.

\section{Acknowledgments}

Ms. Nancy Jackson and Dr. Jeffrey Flynn from Ascension Providence Hospital's Research Department.

\section{Financial statement}

Neither author has any financial disclosures to declare. 


\section{References}

1. Institute of Medicine and National Research Council Committee to Reexamine IOMPWG. The National Academies Collection: Reports funded by National Institutes of Health. In: Rasmussen, KM, Yaktine AL, editors. Weight Gain during Pregnancy: Reexamining the Guidelines. Washington (DC): National Academies Press (US) National Academy of Sciences; 2009.

2. Margerison Zilko CE, Rehkopf D, Abrams B. Association of maternal gestational weight gain with short- and long-term maternal and child health outcomes. Am J Obstet Gynecol. 2010; 202: 574.e1-8. PubMed: https://www.ncbi.nlm.nih.gov/pubmed/20132923

3. Jensen DM, Ovesen P, Beck-Nielsen H, Molsted-Pedersen L, Sorensen $\mathrm{B}$, et al. Gestational weight gain and pregnancy outcomes in 481 obese glucose-tolerant women. Diabetes Care. 2005; 28: 2118-2122. PubMed: https://www.ncbi.nlm.nih.gov/pubmed/16123476

4. Machado LS. Cesarean section in morbidly obese parturients: practical implications and complications. N Am J Med Sci. 2012; 4: 13-18. PubMed: https://www.ncbi.nlm.nih.gov/pubmed/22393542

5. Brost BC, Goldenberg RL, Mercer BM, lams JD, et al. The Preterm Prediction Study: association of cesarean delivery with increases in maternal weight and body mass index. Am J Obstet Gynecol. 1997; 177: 333-337.

PubMed: https://www.ncbi.nlm.nih.gov/pubmed/9290448

6. Tanentsapf I, Heitmann BL, Adegboye AR. Systematic review of clinical trials on dietary interventions to prevent excessive weight gain during pregnancy among normal weight, overweight and obese women. BMC Pregnancy Childbirth. 2011; 11: 81.

PubMed: https://www.ncbi.nlm.nih.gov/pubmed/22029725

7. Curtin SC, Gregory KD, Korst LM, Uddin SF. Maternal Morbidity for Vaginal and Cesarean Deliveries, According to Previous Cesarean History: New Data From the Birth Certificate, 2013. Natl Vital Stat Rep. 2015; 64: 1-13.

PubMed: https://www.ncbi.nlm.nih.gov/pubmed/26046963

8. Clark SL, Hankins GD. Temporal and demographic trends in cerebral palsy-fact and fiction. Am J Obstet Gynecol. 2003; 188: 628-633. PubMed: https://www.ncbi.nlm.nih.gov/pubmed/12634632

9. Menacker F, Curtin SC. Trends in cesarean birth and vaginal birth after previous cesarean. 1991-99. Natl Vital Stat Rep. 2001; 49: 1-16. PubMed: https://www.ncbi.nlm.nih.gov/pubmed/11793867

10. Scheller JM, Nelson KB. Does cesarean delivery prevent cerebral palsy or other neurologic problems of childhood? Obstet Gynecol. 1994; 83: 624-630.

PubMed: https://www.ncbi.nlm.nih.gov/pubmed/8134078

11. Grobman WA, Lai $\mathrm{Y}$, Landon MB, Spong CY, Leveno $\mathrm{KJ}$, et al. Development of a nomogram for prediction of vaginal birth after cesarean delivery. Obstet Gynecol. 2007; 109: 806-812. PubMed: https://www.ncbi.nlm.nih.gov/pubmed/17400840

12. Durnwald CP, Ehrenberg HM, Mercer BM. The impact of maternal obesity and weight gain on vaginal birth after cesarean section success. Am J Obstet Gynecol. 2004; 191: 954-957. PubMed: https://www.ncbi.nlm.nih.gov/pubmed/15467571

13. Juhasz G, Gyamfi C, Gyamfi P, Tocce K, Stone JL. Effect of body mass index and excessive weight gain on success of vaginal birth after cesarean delivery. Obstet Gynecol. 2005; 106: 741-746. PubMed: https://www.ncbi.nlm.nih.gov/pubmed/16199630

14. Hibbard JU, Gilbert S, Landon MB, Hauth JC, Leveno KJ, et al. Trial of labor or repeat cesarean delivery in women with morbid obesity and previous cesarean delivery. Obstet Gynecol. 2006; 108: 125-133. PubMed: https://www.ncbi.nlm.nih.gov/pubmed/16816066

15. Mei JY, Havard AL, Mularz AJ, Maykin MM, Gaw SL, et al. Impact of Gestational Weight Gain on Trial of Labor after Cesarean Success. Am J Perinatol. 2019, 36: 1023-1030.

PubMed: https://www.ncbi.nlm.nih.gov/pubmed/30500964

16. Corp I. IBM SPSS Statistics for Windows. 23 ed: IBM Corp.; 2015.

17. Martin JA, Hamilton BE, Osterman MJK. Births in the United States 2018. NCHS Data Brief. 2019: 1-8.

PubMed: https://www.ncbi.nlm.nih.gov/pubmed/31442195 